\title{
LIE ISOMORPHISMS OF PRIMITIVE RINGS
}

\author{
WALLACE S. MARTINDALE, 3RD
}

1. Introduction. A Lie isomorphism $\phi$ of a ring $R$ onto a ring $R^{\prime}$ is a one-one additive mapping of $R$ onto $R^{\prime}$ which preserves commutators, i.e.,

$$
\begin{aligned}
\phi(x+y) & =\phi(x)+\phi(y), \\
\phi(x y-y x) & =\phi(x) \phi(y)-\phi(y) \phi(x)
\end{aligned}
$$

for all $x, y \in R$. In this paper we study Lie isomorphisms of a primitive ring $R$ onto a primitive ring $R^{\prime}$, where we assume that the characteristic of $R$ is different from 2 and 3 and that $R$ contains three nonzero orthogonal idempotents whose sum is the identity. Such isomorphisms will be shown to be of the form $\sigma+\tau$, where $\sigma$ is either an isomorphism or the negative of an anti-isomorphism of $R$ into a primitive ring $L^{\prime}$ containing $R^{\prime}$ and $\tau$ is an additive mapping of $R$ into the center of $L^{\prime}$ which maps commutators into zero. This generalizes a theorem of Hua [1], who obtained the above result in the case that $R\left(=R^{\prime}=L^{\prime}\right)$ was the ring of all $n \times n$ matrices over a division ring, $n>2$. On the other hand, due to our requirement concerning idempotents, we fall far short of providing a general solution to Herstein's conjecture [2] that the result holds for arbitrary simple rings.

An important part of our proof consists of a repetition of arguments involving matrix units used by Hua [1], and for the sake of completeness (and also because of the relative inaccessability of Hua's paper) we shall reproduce his proofs in some detail when the occasion demands.

The author is especially grateful to Professor Nathan Jacobson for several valuable comments towards improving an earlier version of this paper. In the case of simple rings he indicated how all calculations involving matrix units can be eliminated, and he suggested extending our original result to the more general case of primitive rings.

2. Preliminary results. Let $L$ be the ring of all linear transformations of a (possibly infinite dimensional) vector space $V$ over a division ring $\Delta$. Under the finite topology [3, pp. 248-250] $L$ is a topological ring. Let $S$ be any subset of $L$ and $C=\left\{c_{1}, c_{2}, \cdots, c_{p}\right\}$ a

Presented to the Society, April 29, 1963; received by the editors March 29, 1962. 
finite subset of $L$. We shall say that $S$ satisfies a polynomial identity with respect to $C$ in case there exists a nontrivial polynomial $f(p+q$ noncommutative indeterminates, integral coefficients) such that $f\left(c_{1}, c_{2}, \cdots, c_{p}, x_{1}, x_{2}, \cdots, x_{q}\right)=0$ for all $x_{i} \in S$. As an easy consequence of the above notions we have

Lemma 1. If a subset $S$ of $L$ satisfies a polynomial identity with respect to $C$, then the closure $\mathrm{Cl}(S)$ of $S$ satisfies the same palynomial identity with respect to $C$.

If $x$ and $y$ are elements of a ring, the commutator $x y-y x$ will often be abbreviated by $[x y]$.

Following Hua $[1$, p. 145] we define an element $a$ of $L$ to be an $I$. element in case $a=z+e$, where $z$ lies in the center $Z$ of $L$ and $e$ is an idempotent in $L$. Lemma 1 will enable us to carry over Hua's proof $[1$, p. 146, Theorem 22] of

TheOREm 1. Let $R$ be a dense subring of $L$ of characteristic $\neq 2,3$, and let $a$ be an element of L. Then $a$ is an I-element if and only if

$$
[[[x a] a] a]=[x a] \quad \text { for all } x \in R \text {. }
$$

Proof. If $a$ is an $I$-element it is straight forward to verify that (3) holds. Conversely, if (3) holds, we have by Lemma 1

$$
[[[x a] a] a]=[x a] \quad \text { for all } x \in L
$$

since $\mathrm{Cl}(R)=L$. Relative to a basis for $V$ the elements of $L$ may be considered as row-finite matrices with entries lying in $\Delta$. We can thus write

$$
a=\left(\alpha_{i j}\right), \quad a^{m}=\left(\alpha_{i j}^{(m)}\right), \quad e_{i j}=\text { unit matrix } \in L,
$$

where $i$ and $j$ range over some index set $N$. We set $x=\lambda e_{i j}, \lambda \in \Delta$, and obtain in particular from (4)

$$
\alpha_{i j}^{(2)} \lambda \alpha_{p q}=\alpha_{i j} \lambda \alpha_{p q}^{(2)}, \quad i \neq j, p \neq q
$$

and

$$
\alpha_{i j}^{(3)} \lambda-\alpha_{i j} \lambda=3 \alpha_{i j}^{(2)} \lambda \alpha_{k k}-3 \alpha_{i j} \lambda, \quad i \neq j, \text { all } k .
$$

(5) and (6) hold for all $\lambda \in \Delta$. If $a \notin Z$ there exists a nonsingular matrix $u \in L$ such that $u^{-1} a u$ has a nondiagonal entry different from zero. Since relation (4) and the property of being an $I$-element are preserved under inner automorphisms of $L$, we may assume without loss of generality that $\alpha_{12} \neq 0$. If $\alpha_{p q} \neq 0, p \neq q$, (5) yields

$$
\alpha_{12}^{-1} \alpha_{12}^{(2)} \lambda=\lambda \alpha_{p q}^{(2)} \alpha_{p q}^{-1}
$$


for all $\lambda \in \Delta$. Thus $\alpha_{p q}^{(2)} \alpha_{p q}^{-1}=\beta \in \Phi$, where $\Phi$ is the center of $\Delta$ and $\beta$ is independent of $p$ and $q$, whence

$$
\alpha_{p q}^{(2)}=\beta \alpha_{p q} .
$$

If $\alpha_{p q}=0$, from (5) $\alpha_{p q}^{(2)}=0$, and so (7) is true for all $p \neq q$. Setting $\lambda=1$ in (6) we have

$$
\alpha_{12}^{(3)}-\alpha_{12}=3 \alpha_{12}^{(2)} \alpha_{11}-3 \alpha_{12} \alpha_{11}^{(2)}=3 \alpha_{12}^{(2)} \alpha_{k k}-3 \alpha_{12} \alpha_{k k}^{(2)}
$$

from which we obtain (since char. $\Phi \neq 3$ )

$$
\alpha_{12}^{(2)}\left(\alpha_{11}-\alpha_{k k}\right)=\alpha_{12}\left(\alpha_{11}^{(2)}-\alpha_{k k}^{(2)}\right)
$$

and with the aid of (7)

$$
\alpha_{11}^{(2)}-\alpha_{k k}^{(2)}=\beta\left(\alpha_{11}-\alpha_{k k}\right) .
$$

From (7) and (8) it is easy to see that

$$
a^{2}-\alpha_{11}^{(2)} 1=\beta\left(a-\alpha_{11} 1\right) .
$$

We can then write

$$
\begin{array}{ll}
a^{2}=\beta a+\gamma 1, & \beta \in \Phi, \gamma \in \Delta, \\
a^{3}=\left(\beta^{2}+\gamma\right) a+(\beta \gamma) 1 . &
\end{array}
$$

We choose an $x \in L$ such that $(\gamma 1) x-x(\gamma 1)=0$ but $x a-a x \neq 0$ (e.g., $x=e_{22}$ will work since we are still assuming $\alpha_{12} \neq 0$ ). Now substitution of (9) and (10) in (4) yields

$$
\left(\beta^{2}+4 \gamma\right)(x a-a x)=x a-a x
$$

or

$$
\beta^{2}+4 \gamma-1=0
$$

Using (9) and (11) one verifies that $e=a+\frac{1}{2}(1-\beta) 1$ is an idempotent and thus $a=z+e$, where $z=\frac{1}{2}(\beta-1) 1 \in Z$. Q.E.D.

Another result (and proof) due to Hua [1, p. 155, Theorem 25] we shall find useful is

LeMma 2. Let $R$ be a dense subring of $L$, and let $a$ and $b$ be idempotents of $L$ such that $a b=b a$ and

$$
[[[[x a] b] a] b]+[[x a] b]=0
$$

for all $x \in R$. Then either $a b=0$ or $(1-a)(1-b)=0$. 
Proof. By Lemma 1, (12) holds for all $x \in L$. Multiplication of (12) on the left by $a b$ then gives us

$$
a b x a b-a b x b-a b x a+a b x=0, \quad x \in L .
$$

Factoring, we have $a b x(1-a)(1-b)=0$. Since it is well known that $L$ is a prime ring, either $a b=0$ or $(1-a)(1-b)=0$.

3. Idempotents under Lie isomorphism. Henceforth in this paper we shall suppose that $R$ is a primitive ring of characteristic different from 2 and 3 and containing three nonzero orthogonal idempotents $e_{1}, e_{2}$, and $e_{3}$, whose sum is the identity. We assume further that there exists a Lie isomorphism $\phi$ of $R$ onto a primitive ring $R^{\prime} . R$ will always be treated as a dense subring of the ring $L$ of all linear transformations of a vector space $V$ over a division ring $\Delta$. Similarly we shall consider $R^{\prime}$ as a dense subring of the ring $L^{\prime}$ of all linear transformations of a vector space $V^{\prime}$ over a division ring $\Delta^{\prime}$. It is clear that the characteristic of $R^{\prime}$ must necessarily be different from 2 and 3 . We remark also that if $\phi(x) \in Z^{\prime}$, the center of $L^{\prime}$, then $x \in Z$, the center of $R$.

\section{ThEOREM 2. Either}

(Case 1)

$$
\phi\left(e_{i}\right)=z_{i}+f_{i}
$$

$$
i=1,2,3 \text {. }
$$

or

(Case 2)

$$
\phi\left(e_{i}\right)=z_{i}-f_{i}
$$$$
i=1,2,3,
$$

where $z_{i} \in Z^{\prime},\left\{f_{i}\right\}$ orthogonal idempotents of $L^{\prime}$, and $f_{1}+f_{2}+f_{3}=1$.

Proof. Set $\phi\left(e_{i}\right)=g_{i}$. Since $\left[\left[\left[x e_{i}\right] e_{i}\right] e_{i}\right]=\left[x e_{i}\right]$ for all $x \in R$ and $\phi$ is onto, it follows from (2) that $\left[\left[\left[y g_{i}\right] g_{i}\right] g_{i}\right]=\left[y g_{i}\right]$ for all $y \in R^{\prime}$. By Theorem $1, g_{i}=z_{i}+f_{i}, z_{i} \in Z^{\prime}, f_{i}$ idempotent in $L^{\prime}, i=1,2,3$. A direct calculation shows that $\left[\left[\left[\left[x e_{i}\right] e_{j}\right] e_{i}\right] e_{j}\right]+\left[\left[x e_{i}\right] e_{j}\right]=0$ for all $x \in R$, $i \neq j$. Since $z_{k} \in Z^{\prime}$ we have from (2) that $\left[\left[\left[\left[y f_{i}\right] f_{j}\right] f_{i}\right] f_{j}\right]+\left[\left[y f_{i}\right] f_{j}\right]=0$ for all $y \in R^{\prime}, i \neq j$. Also, from $\left[e_{i} e_{j}\right]=0$, we see that $\left[f_{i} f_{j}\right]=0$. Lemma 2 then says that either $f_{i} f_{j}=0$ or $\left(1-f_{i}\right)\left(1-f_{j}\right)=0$. Suppose $f_{1} f_{2}$ $=f_{1} f_{3}=0$ but $\left(1-f_{2}\right)\left(1-f_{3}\right)=0$. Then $f_{1}=f_{1}\left(1-f_{2}\right)=f_{1}\left(1-f_{2}\right)\left(1-f_{3}\right)$ $=0$, a contradiction. Therefore either $\phi\left(e_{i}\right)=z_{i}+f_{i},\left\{f_{i}\right\}$ orthogonal idempotents, $i=1,2,3$, or $\phi\left(e_{i}\right)=z_{i}+f_{i}=\left(z_{i}+1\right)-\left(1-f_{i}\right)=w_{i}-h_{i}$, $w_{i} \in Z^{\prime},\left\{h_{i}\right\}$ orthogonal idempotents, $i=1,2,3$. Finally, from $0=[x, 1]=\left[x, e_{1}+e_{2}+e_{3}\right]$ for all $x \in R$, we see that $\left[y, f_{1}+f_{2}+f_{3}\right]=0$ for all $y \in R^{\prime}$. By Lemma $1\left[y, f_{1}+f_{2}+f_{3}\right]=0$ for all $y \in L^{\prime}$, and so the idempotent $f_{1}+f_{2}+f_{3}$ lies in $Z^{\prime}$ and must be equal to 1 . 
4. Definition of $\sigma$ and $\tau$. Throughout this section and the next we assume that Case 1 holds, i.e., $\phi\left(e_{i}\right)=z_{i}+f_{i}, i=1,2,3$. We also introduce the notation $R_{i j}=e_{i} R e_{j}, R_{i j}^{\prime}=f_{i} R^{\prime} f_{j}$, and $L_{i j}^{\prime}=f_{i} L^{\prime} f_{j}$. Clearly $R=\oplus \sum_{i, j=1}^{3} R_{i j}$, and, in view of Theorem $2, L^{\prime}=\oplus \sum_{i, j=1}^{3} L_{i j}^{\prime}$. We have made as yet no claim that $R_{i j}^{\prime} \subseteq R^{\prime}$, but in any case $R_{i j}^{\prime}$ is a dense subset of $L_{i j}^{\prime}$.

LEMMA 3. $\phi\left(R_{i j}\right) \subseteq R_{i j}^{\prime}, i \neq j$.

Proof. Let $x \in R_{i j}$ and set $y=\phi(x)$. Since $x=\left[e_{i}\left[x e_{j}\right]\right]$ and $x=\left[e_{i} x\right]$ we have

$$
\begin{aligned}
y & =\left[f_{i}\left[y f_{j}\right]\right]=f_{i} y f_{j}+f_{j} y f_{i}=f_{i}\left(f_{i} y-y f_{i}\right) f_{j}+f_{j}\left(f_{i} y-y f_{i}\right) f_{i} \\
& =f_{i} y f_{j}-f_{j} y f_{i} .
\end{aligned}
$$

Therefore $y=f_{i} y f_{j} \in R_{i j}^{\prime}$, since char. $R^{\prime} \neq 2$.

LEMMA 4. $\phi\left(R_{k k}\right) \subseteq L_{11}^{\prime}+L_{22}^{\prime}+L_{33}^{\prime}$.

Proof. We may assume that $k=1$. Let $x \in R_{11}$ and write $\phi(x)=y$ $=\sum_{i, j=1}^{3} y_{i j}$, where $y_{i j} \in L_{i j}^{\prime}$. From $\left[x e_{2}\right]=\left[x e_{3}\right]=0$ we conclude that $\left[y f_{2}\right]=y_{12}+y_{32}-y_{21}-y_{23}=0$ and $\left[y f_{3}\right]=y_{13}+y_{23}-y_{31}-y_{32}=0$. Hence $y_{i j}=0, i \neq j$, i.e., $y \in L_{11}^{\prime}+L_{22}^{\prime}+L_{33}^{\prime}$.

Since $\phi$ is onto Lemma 3 and Lemma 4 imply

LEMMA 5. $\phi\left(R_{i j}\right)=R_{i j}^{\prime} \subseteq R^{\prime}, i \neq j$.

LEMMA 6. $\phi\left(R_{k k}\right) \subseteq L_{k \mathbf{k}}^{\prime}+Z^{\prime}$.

Proof. Assume $k=1$, let $a \in R_{11}$, and set $b=\phi(a) .[x a]=0$ for all $x \in R_{23}+R_{32}$, and in view of Lemma $5,[y b]=0$ for all $y \in R_{23}^{\prime}+R_{32}^{\prime}$. Now, by Lemma $1,[y b]=0$ for all $y \in \mathrm{Cl}\left(R_{23}^{\prime}+R_{32}^{\prime}\right)=L_{23}^{\prime}+L_{32}^{\prime}$. Since $\mathrm{Cl}\left(L_{23}^{\prime} L_{32}^{\prime}\right)=L_{22}^{\prime}$ and $\mathrm{Cl}\left(L_{32}^{\prime} L_{23}^{\prime}\right)=L_{33}^{\prime}$ we have $[y b]=0$ for all $y \in L_{22}^{\prime}+L_{23}^{\prime}$ $+L_{32}^{\prime}+L_{33}^{\prime}=\left(f_{2}+f_{3}\right) L^{\prime}\left(f_{2}+f_{3}\right)=M$. By Lemma $4, b=b_{1}+c, b_{1} \in L_{11}^{\prime}$, $c \in M$, and so $[y c]=0$ for all $y \in M$. As the center of $M$ is equal to $\left(f_{2}+f_{3}\right) Z^{\prime}$, we can then write $c=\left(f_{2}+f_{3}\right) z, z \in Z^{\prime}$. Therefore we have finally $b=b_{1}+c=\left(b_{1}-f_{1} z\right)+\left(f_{1}+f_{2}+f_{3}\right) z=\left(b_{1}-f_{1} z\right)+z \in L_{11}^{\prime}+Z^{\prime}$.

To summarize our results thus far we have shown that

$$
\begin{aligned}
& \text { if } x \in R_{i j}, \quad i \neq j, \text { then } \phi(x)=x^{*} \in R_{i j}^{\prime}, \\
& \text { if } x \in R_{i i}, \quad \text { then } \phi(x)=x^{*}+z, x^{*} \in L_{i i}^{\prime}, z \in Z^{\prime} .
\end{aligned}
$$

We note that in (14) $x^{*}$ and $z$ are uniquely determined. Indeed, if $\phi(x)=x^{*}+z=y+w, y \in L_{i i}^{\prime}, w \in Z^{\prime}$, then $x^{*}-y \in Z^{\prime} \cap L_{i i}^{\prime}=0$. Hence $y=x^{*}$ and $w=z$. 
Relations (13) and (14) then enable us to define in a natural way a mapping $\sigma$ of $R$ into $L^{\prime}$ according to the rule

$$
\sigma(x)=x^{*}, \quad x \in R_{i j}, \text { all } i, j .
$$

A mapping $\tau$ of $R$ into $Z^{\prime}$ is then defined by

$$
\tau(x)=\phi(x)-\sigma(x), \quad x \in R .
$$

5. Properties of $\sigma$ and $\tau$. We continue to assume that Case 1 holds.

LEMмA 7. $\sigma(x+y)=\sigma(x)+\sigma(y)$ for all $x, y \in R$.

Proof. It suffices to show that $\sigma$ is additive on $R_{k k}$. If $x, y \in R_{k k}$ we have $\sigma(x+y)-\sigma(x)-\sigma(y)=\phi(x+y)-\tau(x+y)-\phi(x)+\tau(x)$ $-\phi(y)+\tau(y)=\tau(x)+\tau(y)-\tau(x+y) \in L_{k \boldsymbol{k}}^{\prime} \cap Z^{\prime}=0$. Thus $\sigma(x+y)=\sigma(x)+\sigma(y)$.

Corollary. $\tau(x+y)=\tau(x)+\tau(y), x, y \in R$.

LeMma 8. $\tau(x y-y x)=0$ for all $x, y \in R$.

Proof. Suppose $x \in R_{12}$. If $y \in R_{i j},(i, j) \neq(2,1)$, then $x y-y x \in R_{p q}$, $p \neq q$, and $\tau(x y-y x)=0$. If $y \in R_{21}, \tau(x y-y x)=\phi(x y-y x)-\sigma(x y-y x)$ $=\phi(x) \phi(y)-\phi(y) \phi(x)-\sigma(x y-y x)=\sigma(x) \sigma(y)-\sigma(y) \sigma(x)-\sigma(x y-y x)$ $\in L_{11}^{\prime}+L_{22}^{\prime}$. Since $\tau(x y-y x) \in Z^{\prime}$, it follows that $\tau(x y-y x)=0$. In general, if $x \in R_{i j}, i \neq j$, then $\tau(x y-y x)=0$ for all $y \in R$.

Next suppose that $x \in R_{11}$. We have already shown that if $y \in R_{i j}$, $i \neq j$, then $\tau(x y-y x)=0$. Clearly $\tau(x y-y x)=0$ in case $y \in R_{22}$ or $y \in R_{33}$. If $y \in R_{11}$, we have $\tau(x y-y x)=\phi(x y-y x)-\sigma(x y-y x)$ $=\sigma(x) \sigma(y)-\sigma(y) \sigma(x)-\sigma(x y-y x) \in L_{11}^{\prime}$. Since $\tau(x y-y x) \in Z^{\prime}$, $\tau(x y-y x)=0$. The same argument holds in case $x \in R_{22}$ or $x \in R_{33}$. We conclude that $\tau(x y-y x)=0$ for all $x, y \in R$.

Corollary. $\sigma(x y-y x)=\sigma(x) \sigma(y)-\sigma(y) \sigma(x)$ for all $x, y \in R$.

Lemma 9. Let $x \in R_{i j}, i \neq j$, and $y \in R$. Then $\sigma(x y)=\sigma(x) \sigma(y)$ and $\sigma(y x)=\sigma(y) \sigma(x)$.

Proof. We may assume that $x \in R_{12}$. For $y \in R_{12}$, we have $\sigma(x y)$ $=\sigma(0)=0=\sigma(x) \sigma(y)$ and, similarly, $\sigma(y x)=\sigma(y) \sigma(x)$. Thus suppose $y \in R_{i j},(i, j) \neq(1,2)$. Then $x y \in R_{1 j}, \sigma(x) \in L_{12}^{\prime}, \sigma(y) \in L_{i j}^{\prime}$, and so we see that both $\sigma(x y)$ and $\sigma(x) \sigma(y)$ are elements of $L_{1 j}^{\prime}$. Similarly $\sigma(y x)$ and $\sigma(y) \sigma(x)$ lie in $L_{i 2}^{\prime}$. Using the corollary to Lemma 8 we have $\sigma(x y)-\sigma(y x)=\sigma(x y-y x)=\sigma(x) \sigma(y)-\sigma(y) \sigma(x)$. Therefore $\sigma(x y)$ $-\sigma(x) \sigma(y)=\sigma(y x)-\sigma(y) \sigma(x) \in L_{1 j}^{\prime} \cap L_{i 2}^{\prime}=0$ and the conclusion follows.

TheOREM 3. $\sigma$ is an isomorphism of $R$ into $L^{\prime}$. 
PROOF. In order to verify that $\sigma(x y)=\sigma(x) \sigma(y)$ for all $x, y \in R$, we need only, in view of Lemma 9, consider the case where $x \in R_{11}$ and $y \in R_{k k}$. If $y \in R_{22}$ or $y \in R_{33}$ we have $\sigma(x y)=0=\sigma(x) \sigma(y)$. Thus assume $y \in R_{11}$. If $r \in R_{21}$, repeated use of Lemma 9 enables us to write $\sigma(r)[\sigma(x y)-\sigma(x) \sigma(y)]=\sigma(r) \sigma(x y)-\sigma(r) \sigma(x) \sigma(y)=\sigma(r x y)-\sigma(r x) \sigma(y)$ $=\sigma(r x y)-\sigma(r x y)=0$. Hence $R_{21}^{\prime}[\sigma(x y)-\sigma(x) \sigma(y)]=0$, and, by Lemma 1, $L_{21}^{\prime}[\sigma(x y)-\sigma(x) \sigma(y)]=0$. Since $f_{1} \in L_{11}^{\prime}=\operatorname{Cl}\left(L_{12}^{\prime} L_{21}^{\prime}\right)$ we have $\sigma(x y)$ $-\sigma(x) \sigma(y)=f_{1}[\sigma(x y)-\sigma(x) \sigma(y)]=0$, i.e., $\sigma(x y)=\sigma(x) \sigma(y)$.

It remains to show that $\sigma$ is one-one. Suppose $\sigma(x)=0$ for some $x \in R$. Then $\sigma(x y)=\sigma(x) \sigma(y)=0$ for all $y \in R$, and so $\phi(x y)=\tau(x y) \in Z^{\prime}$. It follows that $x y \in Z$ and in particular that $x \in Z$ and $x e_{1} \in R_{11} \cap Z$. Thus $x e_{1}=0$, and, since $x \in Z, x=0$.

6. Treatment of Case 2. In this section we assume that Case 2 holds, that is, $\phi\left(e_{i}\right)=z_{i}-f_{i}, i=1,2,3$ (see Theorem 2). Let $\theta$ be an anti-isomorphism of $L^{\prime}$ onto a ring $L^{\prime \prime}$ (such exists). Then $-\theta$ is easily seen to be a Lie isomorphism of $L^{\prime}$ onto $L^{\prime \prime}$, whence $\psi=-\theta \phi$ is a Lie isomorphism of $R$ onto a subring $R^{\prime \prime}$ of $L^{\prime \prime}$. Furthermore $\psi\left(e_{i}\right)=-\theta\left(z_{i}-f_{i}\right)=-\theta\left(z_{i}\right)+\theta\left(f_{i}\right)=w_{i}+g_{i}, \quad\left\{g_{i}\right\}$ orthogonal idempotents of $L^{\prime \prime}$. Since Case 1 arguments apply to $\psi$ we have by Theorem 3 and Lemma 8 that $\psi=\sigma+\tau$, where $\sigma$ is an isomorphism of $R$ into $L^{\prime \prime}$ and $\tau$ is an additive mapping of $R$ into $Z^{\prime \prime}$ which maps commutators into zero. Hence $-\theta \phi=\sigma+\tau$, from which we obtain $\phi=-\theta^{-1} \sigma-\theta^{-1} \tau=\sigma^{\prime}+\tau^{\prime}$, where $\sigma^{\prime}$ is the negative of an anti-isomorphism of $R$ into $L^{\prime}$ and $\tau^{\prime}$ is again an additive mapping of $R$ into $Z^{\prime}$ which maps commutators into zero.

7. The main result and special cases. From Lemma 8, Theorem 3, and the discussion of the preceding section it is clear that we have completed the proof of

TheOREM 4. Let $\phi$ be a Lie isomorphism of a primitive ring $R$ onto a primitive ring $R^{\prime}$, where the characteristic of $R$ is different from 2 and 3 and $R$ contains three nonzero orthogonal idempotents whose sum is the identity. Then $\phi$ is of the form $\sigma+\tau$, where $\sigma$ is either an isomorphism or the negative of an anti-isomorphism of $R$ into a primitive ring $L^{\prime}$ containing $R^{\prime}$ and $\tau$ is an additive mapping of $R$ into the center of $L^{\prime}$ which maps commutators into zero.

Consider now the special case of Theorem 4 in which $R$ and $R^{\prime}$ are simple. Using again notation introduced earlier and for simplicity assuming Case 1 , we point out that the subring of $R$ generated by the union of the $R_{i j}, i \neq j$, is actually an ideal of $R$ and hence equal to $R$. Since $\sigma$ is an isomorphism and $\sigma\left(R_{i j}\right) \subseteq R^{\prime}, i \neq j$, it follows that 
$\sigma(R) \subseteq R^{\prime}$. Similarly the subring generated by the union of the $R_{i j}^{\prime}$, $i \neq j$, is an ideal of $R^{\prime}$ and thus equal to $R^{\prime}$. Since $\sigma\left(R_{i j}\right)=R_{i j}^{\prime}, i \neq j$, we conclude that $\sigma(R)=R^{\prime}$. We have thereby proved

TheOREM 5. Let $\phi$ be a Lie isomorphism of a simple ring $R$ onto a simple ring $R^{\prime}$, where the characteristic of $R$ is different from 2 and 3 and $R$ contains three nonzero orthogonal idempotents whose sum is the $i d e n t i t y$. Then $\phi$ is of the form $\sigma+\tau$, where $\sigma$ is either an isomorphism or the negative of an anti-isomorphism of $R$ onto $R^{\prime}$ and $\tau$ is an additive mapping of $R$ into the center of $R^{\prime}$ which maps commutators into zero.

We conclude by illustrating Theorem 4 with an example of a Lie automorphism $\phi$ of a primitive ring $R$ such that $\sigma(R)$ is not contained in $R$. Let $\Delta$ be the field $\Phi(x, y), \Phi$ the field of rationals, and let $\Omega$ be the subfield $\Phi(x)$. We take $R$ to be the ring of all countably infinite matrices of the form $A+D$, where $A=\left(a_{i j}\right), a_{i j} \in \Delta$, only a finite number of the $a_{i j}$ unequal to zero, and $D=\operatorname{diag}\{d, d, d, \cdots\}, d \in \Omega$. If $a_{i j}=f_{i j}(x, y)$ we let $\bar{a}_{i j}=f_{i j}(y, x)$. We define a mapping $\phi$ of $R$ onto itself by

$$
\phi(A+D)=\bar{A}+D
$$

where $\bar{A}=\left(\bar{a}_{i j}\right)$. $\phi$ is easily seen to be a Lie automorphism of $R$ and may be written in the form $\sigma+\tau$ according to Theorem 4. One may verify that $\sigma(A)=\bar{A}$ for all $A$. Now let $D=\operatorname{diag}\{x, x, x, \cdots\} . \sigma(D)$ must then be of the form $\operatorname{diag}\{c, c, c, \cdots\}$. If $A=\operatorname{diag}\{x, 0,0, \cdots\}$ then $\sigma(A)=\operatorname{diag}\{y, 0,0, \cdots\}$. From $\sigma(A) \sigma(D)=\sigma(A D)=\sigma\left(A^{2}\right)$ we obtain $\operatorname{diag}\{y c, 0,0, \cdots\}=\operatorname{diag}\left\{y^{2}, 0,0, \cdots\right\}$. It follows that $c=y$ and $\sigma(D)=\operatorname{diag}\{y, y, y, \cdots\}$. Hence $\sigma(R) \Phi R$.

\section{BIBLIOGRAPHY}

1. L. Hua, $A$ theorem on matrices over an sfield and its applications, J. Chinese Math. Soc. (N.S.) 1 (1951), 110-163.

2. I. N. Herstein, Lie and Jordan structures in simple associative rings, Bull. Amer. Math. Soc. 67 (1961), 517-531.

3. N. Jacobson, Lectures in abstract algebra, Vol. 2, Van Nostrand, New York, 1953.

\section{Smith College}

